\title{
Inhibition of the mammalian target or rapamycin (mTOR): a potential therapeutic strategy for multiple system atrophy
}

\author{
Alain Ndayisaba ${ }^{1} \cdot$ Gregor K. Wenning $^{1}$
}

Received: 24 December 2019 / Accepted: 25 December 2019 / Published online: 9 January 2020

(c) Springer-Verlag GmbH Germany, part of Springer Nature 2020

Multiple system atrophy (MSA) is an aggressive neurodegenerative disorder characterized by the deposition of aggregates containing $\alpha$-synuclein ( $\alpha$-syn) in oligodendrocytes and neurons. The disease is relentlessly fatal, and most patients die within 9 years of disease onset. While the events that trigger the presence and accumulation of $\alpha$-syn in oligodendrocytes and neurons remains a mystery, the failure of intracellular protein clearance mechanisms appears to be a major pathophysiology pathway.

Pathways involved in autophagy and lysosomal function have received increased attention because their modulation could be a potential therapeutic strategy for neurodegenerative proteinopathies. One of such pathways is the mammalian target of rapamycin complex (mTOR) signaling pathway. Activation of the mTOR pathway promotes cell survival, growth and proliferation via increased protein synthesis, whereas inhibition of the mTOR pathway results in increased autophagy, among many other biological effects (Fig. 1). Thus, it is tempting to hypothesize that increased activation of autophagy via mTOR inhibition may result in reduced proteinopathy-related neurodegeneration $[1,3]$.

In keeping with this hypothesis, treatment with rapamycin (also known a sirolimus, a potent inhibitor of the mTOR pathway) in animal models of synucleinopathy reduced the amount of intracellular aggregated $\alpha$-syn [6]. Similarly, treatment with rapamycin for 16 weeks in a transgenic mouse model of MSA overexpressing human $\alpha$-syn exclusively in oligodendrocytes resulted in decreased $\alpha$-syn levels in substantia nigra pars compacta, but not in striatum. Importantly, mice treated with sirolimus developed no motor improvement, consistent with decreased numbers of

Gregor K. Wenning

gregor.wenning@i-med.ac.at

1 Division of Clinical Neurobiology, Department of Neurology, Medical University of Innsbruck, Anichstraße 35, 6020 Innsbruck, Austria tyrosine hydroxylase-positive neurons similar to untreated MSA mice. Yet, the overall number of Nissl positive neurons was comparable to healthy control mice suggesting that rapamycin exerts some degree of neuroprotection [8, 13]. On the other hand, inhibition of autophagy may not represent a primary or essential event in the pathogenesis of MSA since inhibition of macroautophagy via bafilomycin A1 did not promote $\alpha$-syn aggregation in oligodendroglial cell lines and transgenic mice overexpressing human wild type $\alpha$-syn [5, 6].

But the effects of mTOR inhibition go beyond the activation of autophagy and inhibition of apoptosis. Leigh syndrome is a severe neurological disease caused by mitochondrial dysfunction due to mutations in mitochondrial DNA or deficiency of pyruvate dehydrogenase. It manifests with neurological and psychomotor deficits with patients dying typically before age 3 [4]. In a recent study, treatment of cultured neurons obtained from patients with Leigh syndrome with rapamycin resulted in decreased energy consumption, presumably via a decrease of protein synthesis, which prevented glutamate toxicity [14]. Since mitochondrial dysfunction constitutes a central aspect in MSA pathogenesis [10], treatment with rapamycin in MSA may also have beneficial effects through mitochondrial pathways.

Patients with MSA also appear to have dysregulation of the upstream insulin/insulin-like growth factor (IGF)-1 pathway [12]. Putaminal neurons and oligodendrocytes of mice with MSA had increased expression of the insulin receptor substrate 1 phosphorylated at serine residue 312 , which is a marker of insulin resistance, compared to neurons and oligodendrocytes from other, less affected brain areas in MSA, such as the temporal cortex [2]. Since IGF-1, also promotes mTOR activation via the PI3-K/Akt pathway (Fig. 1), mTOR inhibition with rapamycin/sirolimus may partially block the deleterious effects of excessive insulin and IGF-1 levels.

In summary, multiple sources of evidence support the rationale to test if rapamycin/sirolimus can effectively block 


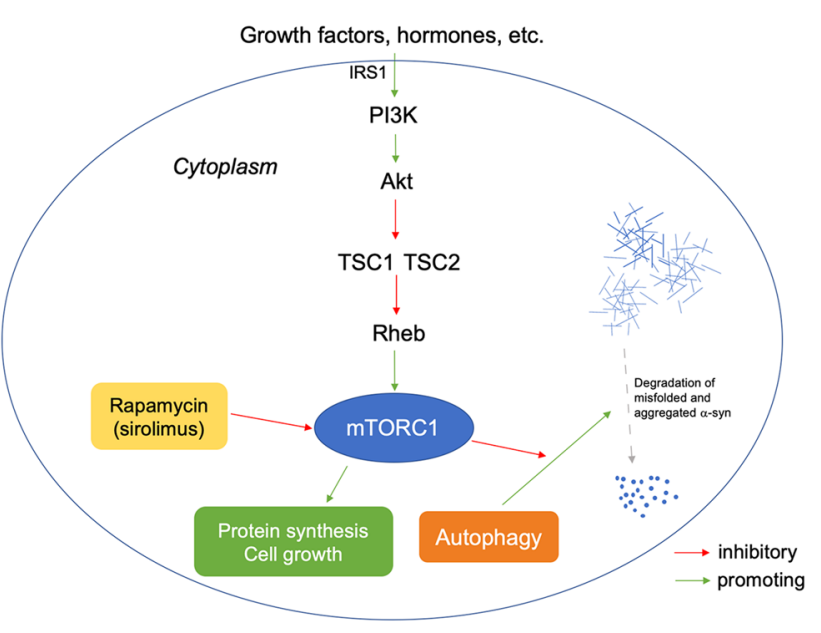

Fig. 1 Simplified organization of the mTOR complex 1 (mTORC1) pathway. Rapamycin/sirolimus inhibits mTORC1 resulting in enhanced clearance and autophagy of intracellular proteins, such as alpha-synuclein. $\alpha$-syn alpha-synuclein, IRS1 insulin receptor substrate type $1, P I 3 K$ phosphoinositide 3-kinase, Akt serine/threonine kinase, TSC tuberous sclerosis complex, $m$ TORC mammalian target of rapamycin complex

the mTOR pathway and enhance the autophagy of $\alpha$-syn (Fig. 1). Sirolimus has been approved by most regulatory agencies since the 1990s as an immunosuppressant to prevent organ transplant rejection, and its profile of adverse events is very well known. An ongoing single-center phase-2 double-blind randomized placebo-controlled trial at New York University (NYU), led by Drs. Palma and Kaufmann, aims to determine whether sirolimus is of sufficient promise to slow the progression of MSA (ClinicalTrials.gov: NCT03589976). In this trial, patients receive oral sirolimus at dosages ranging from 2 to $6 \mathrm{mg} /$ day, or matching placebo, for a year [11]. These dosages are well tolerated and associated with relatively low immunologic risk [7, 9]. The trial will be completed by late 2021 and first results are expected by early 2022 . Since we are far from understanding the cause of this devastating disease, trials like this one with sirolimus are much needed. We owe it to all patients and their relatives in dire need for treatments.

\section{Funding None.}

\section{Compliance with ethical standards}

Conflict of interest The authors declare that they have no conflict of interest.

\section{References}

1. Aso E, Ferrer I (2013) It may be possible to delay the onset of neurodegenerative diseases with an immunosuppressive drug (rapamycin). Expert Opin Biol Ther 13:1215-1219

2. Bassil F, Canron MH, Vital A, Bezard E, Li Y, Greig NH, Gulyani S, Kapogiannis D, Fernagut PO, Meissner WG (2017) Insulin resistance and exendin-4 treatment for multiple system atrophy. Brain 140:1420-1436

3. Bove J, Martinez-Vicente M, Vila M (2011) Fighting neurodegeneration with rapamycin: mechanistic insights. Nat Rev Neurosci 12:437-452

4. Chen L, Cui Y, Jiang D, Ma CY, Tse HF, Hwu WL, Lian Q (2018) Management of Leigh syndrome: current status and new insights. Clin Genet 93:1131-1140

5. Fellner L, Buchinger E, Brueck D, Irschick R, Wenning GK, Stefanova N (2018) Limited effects of dysfunctional macroautophagy on the accumulation of extracellularly derived $\alpha$-synuclein in oligodendroglia: implications for MSA pathogenesis. BMC Neurosci $19: 32$

6. Klucken J, Poehler AM, Ebrahimi-Fakhari D, Schneider J, Nuber S, Rockenstein E, Schlötzer-Schrehardt U, Hyman BT, McLean PJ, Masliah E, Winkler J (2012) Alpha-synuclein aggregation involves a bafilomycin A 1-sensitive autophagy pathway. Autophagy 8:754-766

7. Kraig E, Linehan LA, Liang H, Romo TQ, Liu Q, Wu Y, Benavides AD, Curiel TJ, Javors MA, Musi N, Chiodo L, Koek W, Gelfond JAL, Kellogg DL (2018) A randomized control trial to establish the feasibility and safety of rapamycin treatment in an older human cohort: immunological, physical performance, and cognitive effects. Exp Gerontol 105:53-69

8. Lopez-Cuina M, Guerin P, Bezard E, Meissner W, Fernagut PO (2018) Rapamycin for treating MSA: a preclinical proof of concept study. Mov Disord 33:S441

9. Mannick JB, Del Giudice G, Lattanzi M, Valiante NM, Praestgaard J, Huang B, Lonetto MA, Maecker HT, Kovarik J, Carson S, Glass DJ, Klickstein LB (2014) mTOR inhibition improves immune function in the elderly. Sci Transl Med 6:268ra179

10. Monzio Compagnoni G, Di Fonzo A (2019) Understanding the pathogenesis of multiple system atrophy: state of the art and future perspectives. Acta Neuropathol Commun 7:113

11. Palma JA, Martinez J, Barnes E, Simon S, Norcliffe-Kaufmann L, Kaufmann H (2019) A futility trial of sirolimus in multiple system atrophy: protocol, recruitment and preliminary adverse event profile. Neurology 92:P3.8-019

12. Pellecchia MT, Pivonello R, Longo K, Manfredi M, Tessitore A, Amboni M, Pivonello C, Rocco M, Cozzolino A, Colao A, Barone $\mathrm{P}$ (2010) Multiple system atrophy is associated with changes in peripheral insulin-like growth factor system. Mov Disord 25:2621-2626

13. Refolo V, Bez F, Polissidis A, Kuzdas-Wood D, Sturm E, Kamaratou M, Poewe W, Stefanis L, Angela Cenci M, Romero-Ramos M, Wenning GK, Stefanova N (2018) Progressive striatonigral degeneration in a transgenic mouse model of multiple system atrophy: translational implications for interventional therapies. Acta Neuropathol Commun 6:2

14. Zheng X, Boyer L, Jin M, Kim Y, Fan W, Bardy C, Berggren T, Evans RM, Gage FH, Hunter T (2016) Alleviation of neuronal energy deficiency by mTOR inhibition as a treatment for mitochondria-related neurodegeneration. Elife 5:pii: e13378 